\title{
La función del dialecto de Walter Morel en Sons and Lovers y claves de su traducción al español
}

\author{
Luis Javier Conejero Magro \\ Universidad de Extremadura \\ conejero.lj@gmail.com
}

\begin{abstract}
https://dx.doi.org/10.12795/futhark.2014.i9.03
Abstract: The purpose of this essay is to examine and identify the functions of the East Midlands dialect in Sons and Lovers in order to explore the possibilities of its translation into Spanish. The fact that this regional variety of English also expresses a deep conflict of the working and the upper classes in this novel, makes it possible for the linguistic contrast, if not conflict, between Gertrude and Walter Morel to become patent in the target text, too. Indeed, by replacing the East Midlands dialect with a nonstandard variety of Spanish, not identified with the world of crime and marginalisation, one may find a way to partially translate a local dialect that, per se, is always an untranslatable one.
\end{abstract}

Key words: D.H. Lawrence, Sons and Lovers, translation, dialect, sociolinguistics.

Resumen: Este artículo examina e identifica las funciones del dialecto de los East Midlands en la obra Sons and Lovers para explorar posteriormente las posibilidades de su traducción en español. El hecho de que dicha variedad regional de la lengua inglesa exprese un conflicto entre los trabajadores y la clase alta en esta novela obliga a que el conflicto lingüístico se vea reflejado en el texto de llegada también. Por lo tanto, reemplazando el dialecto de los East Midlands por una variedad no-estándar del español, que no se identifique con el mundo de la delincuencia, se podría encontrar 
66

una solución parcial para traducir un dialecto local que es en si un intra.
ducible

Palabras clave: D.H. Lawrence, Sons and Lovers, traducción, dialecto, so. ciolingüistica.

A nadie se le oculta que, en principio, la traducción de una obra literaria escrita en una variedad dialectal del inglés a otra lengua no tendria por qué plantear ni más ni menos problemas que la de cualquier texto literario cuyo vehículo narrativo sea el inglés estándar. La dificultad, como se sabe, surge, y se incrementa a veces hasta alcanzar niveles insospechados, cuando se trata de trasladar a otro idioma fragmentos dialectales incrustados en una obra literaria cuya base textual no es el inglés dialectal sino el estándar. Esto es fácil de comprender si se tiene en cuenta que, cuando aparece un texto dialectal dentro de una obra escrita en lenguaje estándar, aquél suele desempenar una función muy concreta o constituir una marca distintiva dentro del entramado narrativo. De ahí que deba mantenerse en la lengua de llegada. Es decir, cuando se da esta circunstancia, se produce en la obra literaria una relación muy peculiar entre la norma dialectal y la norma estándar; y esa relación cumple siempre una función, pues forma parte de la estructura significativa de la obra. Por eso inevitablemente se resiente la calidad del texto literario cuando se desdibuja - desaparece esa diferencia, aun cuando la función del personaje de expresión dialectal, sea éste de naturaleza individual o colectiva, se reduzca únicamente a añadir un toque costumbrista o simplemente un sabor local. La necesidad de que esta función diferenciadora se mantenga en la lengua a la que se traduce el texto es aún mayor, por supuesto, cuando la razón del empleo del dialecto tiene que ver con la representación de esa segregación linguístico-cultural a que, a causa de viejos y manidos prejuicios, cuando no estigmas, se suelen ver sometidos sus usuarios. Y esto, como es sabido, se revela como una constante en la literatura de habla inglesa, como probablemente ocurre también en las de otros idiomas, a partir de la consolidación del estándar como lengua del estado nacional moderno.

Una fuente de ejemplos frecuentes y elocuentes en este sentido, la hallamos en esa interminable galería de personajes literarios que, sobre todo a partir del despertar renacentista y tanto en obras de tea- 
tro como en novelas de todo tipo, han sido objeto de la mofa de los espectadores o la hilaridad del público lector, sin haber hecho otra "gracia" que la de expresarse en su propia modalidad linguística. Otra cantera abundante en casos paradigmáticos, y dentro también de la creación literaria, la constituye la configuración de isoglosas geolectales o sociolectales que contribuyen a establecer o reforzar el sentido de pertenencia o identidad de un grupo social, o incluso de todo un pueblo, frente a una comunidad linguística dominante. Es decir, esos textos en que la utilización del dialecto expresa la afirmación o reafirmación de una idiosincrasia cultural y lingüística de un grupo minoritario o toda una sociedad sometidos. Parece, por tanto, innecesario insistir en que, en estos supuestos, el traductor ha de tener muy presente el sentido que tiene el dialecto en la obra de partida e intentar reproducirlo en el texto terminal. En efecto, la naturaleza de la correspondencia de un pasaje dialectal del texto original en el traducido variará según ese sentido o, para decirlo de otro modo, según la función que cumpla en su propio contexto literario. De ahí que, antes de acometer la tarea de traducir una obra de estas características, resulte imprescindible un análisis detallado tanto de la personalidad del hablante dialectal como del papel que éste desempeña en el conjunto de dicha obra y, por supuesto, del sentido específico de cada una de sus intervenciones.

Todo esto, como se puede deducir fácilmente, no es sino la aplicación concreta de un principio general de la traducción literaria, a saber, no es posible el logro de una traducción medianamente fiable sin un ejercicio previo de crítica literaria; y, más concretamente, sin un análisis textual pormenorizado que nos ayude a descubrir la naturaleza de esos elementos lingüísticos y estilísticos en torno a los que se suele configurar la obra de creación. Sólo de este modo será posible la identificación de las múltiples funciones y efectos de los referidos elementos en el texto. En consecuencia, la identificación de los recursos retóricos y los matices estilístico-textuales de una obra -que suelen incluir, entre otros, aspectos tan dispares como los tipos de estructura morfosintáctica predominantes o la variedad de áreas léxicas y registros- supone un paso de importancia decisiva, si no imprescindible, en el proceso de traducción.

Futhark 9 (2014)

Conejero Magro, La función del dialecto de Walter Morel, 65-84

ISSN 1886-9300 
En la obra de D.H. Lawrence hallamos ejemplos muy elocuentes de lo que se acaba de decir, pues constituye toda una cantera de personajes de expresion dialectal o cuya habla fluctua entre el estandar y el dialecto; y, lo que es mas importante aún, en algunas de sus novelas y narraciones cortas se dan intervenciones dialectales con las funciones más diversas. Entre esos personajes destaca sin duda Walter Morel pues el lenguaje que utiliza tiene mucho que ver con la complejidad de su situacion social y familiar, y esta es fruto, en buena medida, del conflicto de clase y cultural que se libra en su propio hogar'.

No se pretende aquí ofrecer un analisis exhaustivo del componente dialectal de Sons and Looers. Ni siquiera se intenta explicar su funcion en todos $\mathrm{y}$ cada uno de los personajes de expresión dialectal en esta obra de Lawrence. Ello rehasaria el marco necesariamente limitado de este ensayo y, sobre todo, resultaría innecesario dada la calidad de los trabajos que se han hecho sobre este tema y la abundante crítica exis-

i Huelga decir que la diferencia social, cultural y lingüística que separa a Walter Morel de su esposa tiene mucho que ver con el entramado estilístico de la obra. Así, mientras ella se expresa siempre en el inglés estándar de su clase, Walter Morel se aferra a la variedad local del dialecto de los East Midlands. Sobre la base social de esa diferencia en canto a la expresión lingüistica ofrecen análisis muy atinados José Mateo Martinez en su libro Mundos en conflicto: Estudio lingüístico-ideológico de 'Sons ard Lovers' de D.H. Lawrence (Alicante: Secretariado de Publicaciones Universidad de Alicante, 1992), sobre todo en las páginas 97 y 98; y Azer Banu Kemaloglu y Sercan Hamza Baglama en "A Victorian Extension of Sons and Lovers: a Marxist Perspective", publicado en European Academic Research (1. 10. [Jan. 2014]), especialmente en las páginas 3326 y 3347 . Todo esto tiene unas consecuencias de caracter literario que Mortad-Serir Ilhem sintetiza de modo muy elocuente: “[in] the story of class differences of married couple where the husband speaks local dialect in contrast to his wife's refined English, Lawrence sets authentic scenes in Nottingham coalfields found in the rolling hills and coal-pitted region known as the British Midlands to interweave the plot and the theme in his novel Sons and Lovers that brought fresh realism to literatura at the turn of twentieth century;" (Intemational Joumal of English Language and Literature Studies, 2. 2. 2013: 118). También Nozar Niazi establece una clara relación entre los dos niveles de habla que dialogan o se enfrentan en Sons and Lovers y su entramado estilístico, aunque casi pasa por alto un tema tan importante como el conflicto de clase que se entabla en esta obra para destacar, hasta niveles que alcanzan la idealización, aspectos de menos importancia en esta historia como, por ejemplo, su dimensión sensual o espiritual. Véase su "A Stylistic Analysis of D. H. Lawrence's 'Sons and Lovers'" (Australian Intermational Academic Centre, 2. 4. Uuly 2013]). 
tente sobre la novela ${ }^{2}$. Se trata tan solo de recordar, a título de ejemplo, algunos rasgos del dialecto de los East Midlands que emplea Walter Morel que el traductor ha de tener presentes, pues nos ayudan a identificar la función y valorar la importancia que tiene el habla de este personaje en el entramado formal y argumental del relato ${ }^{3}$. Esto nos permitiría, sin duda alguna, aproximarnos al sentido total de la obra y, en consecuencia, nos puede dar algunas claves de gran utilidad a la hora de evaluar las traducciones existentes o abordar una nueva ${ }^{4}$.

\footnotetext{
2 Véase Richard Leith, "Dialogue and Dialect in D. H. Lawrence" (Style 14 [1980]: pp. 245-58); Yingly Liu y Xiufeng Jin, "The Unique Language Style in Sons and Lovers" (US China Foreign Language 4. 3. [2006]: pp. 5-9); Consuelo Montes Granado, D. H. Lawrence: el dialect en sus novelas (Salamanca: Ediciones Universidad de Salamanca, 1990), "Los pronombres personales con especial mención de los usos de thou y you en los personajes dialectales de la obra de D. H. Lawrence" (Studia Zamorensia 8 [1987]: pp. 141-57); “Un fenómeno sociolingüistico y su ilustración en el temprano devenir linguiústico de D. H. Lawrence" (Revista Alicantina de Estudios Ingleses 3 [1990]: pp. 107-13); entre otros.
}

3 Deseo expresar mi agradecimiento a María Teresa Corchado Pascasio por haberme permitido consultar el manuscrito de un estudio suyo sobre la narración corta de D. H. Lawrence aún inédito. Ello me ha permitido contrastar algunas de las ideas que aquí se exponen con sus conclusiones sobre las historias cortas incluidas en su trabajo $y$, en no pocas ocasiones, reafirmarme en algunas de las hipótesis de partida.

4 Para la realización de esa nueva traducción que aún espera la recepción de Sons and Lovers en español, resulta de gran utilidad el excelente artículo de Luisa María González Rodríguez "Reflexiones sobre la traducción al español de los parlamentos dialectales en la novela Sons and Lovers de D.H. Lawrence" (Hermēneus: Revista de Traducción e Interpretación, 13 [2011]: pp. 1-12). En ese ensayo, sobre todo en las páginas 1,2 y 3 , se incluye un resumen muy pertinente sobre la complejidad del traslado a otra lengua de textos dialectales en contextos en que éstos se contraponen al estándar, así como las dificultades que plantea este tipo de traducción. Sin embargo, aunque González Rodríguez se refiere continuamente a los valores de tipo social y cultural, y a las preferencias y la conducta de Walter Morel y su esposa, Gertrude Coppard, su análisis se detiene tal vez excesivamente en la dimensión individual del conflicto, lo que hace que inevitablemente se resienta el perfil de ese colectivo que también encarna cada uno de los dos cónyuges. En efecto, la obra no sólo gira en torno a las diferencias que separan a esos dos seres individuales e irrepetibles de la historia que nos cuenta Lawrence, sino también en torno a un conflicto que transciende lo individual y es de 
Para empezar, conviene identificar alguna de esas intervenciones del personaje minero en que su lenguaje fluctua entre la variedad regional de Nottinghamshire y un uso mas o menos estandarizado del ingles, y no solo por la mayor o menor carga dialectal de sus frases sino, sobre todo, como resultado de cambios súbitos del dialecto al estándar, o viceversa. Es preciso prestar una atención especial a esos pasajes, pues este tipo de oscilación suele responder a momentos más o menos críticos de su relación con su esposa o los demás miembros de la familia, por lo que se revela como una marca inconfundible del ritmo emotivo de sus vivencias más intimas. No hay que olvidar, en ese sentido, que el núcleo familiar en torno al que se configura Sons and Looers se ve amenazado por una confrontación de valores y aspiraciones de naturaleza social y cultural; y que esta pugna es en buena medida la base del conflicto entre Walter Morel y su cónyuge o sus hijos. De ese conflicto se nutre precisamente la tensión familiar, sociocultural y hasta linguística que se detecta bajo la estructura argumental de la obra. De ahí que el tratamiento del dialecto en la traducción sea un factor decisivo para garantizar el grado de fidelidad y coherencia exigible al texto de llegada.

El primer pasaje elegido es ese diálogo entre Walter Morel y Gertrude Coppard en el que las intervenciones del personaje minero se realizan, también por primera vez, en un lenguaje estándar o muy próximo al estándar. La razón de su opción momentánea por este nivel de habla, que es un ejemplo antológico de "deferencia" linguiística, se explica en la novela unas líneas más arriba:

She was to the miner that thing of mystery and fascination, a lady. When she spoke to him, it was with a southern pronunciation and a purity of English which thrilled him to hear. ${ }^{5}$

Esa concesión del minero, muy excepcional por cierto, hace, en efecto, que en las primeras palabras que se intercambian apenas haya diferencia entre el habla utilizado por Gertrude, su interlocutora y

dimensión histórica; y esto, logicamente, también tiene que ver con la solución que se dé al traslado del dialecto a otra lengua.

5 Lawrence, D.H., Sons and Lovers (Oxford University Press, 1985, p.15). A partir de ahora todas las referencias textuales a Sons and Lovers corresponderán a esta edición y la página se indicará en el texto entre paréntesis.

Futhark 9 (2014)

ISSN 1886-9300 
futura esposa, y la del minero. Por lo tanto, ese breve dialogo se inscribe casi totalmente en la norma generalmente aceptada. He aquí las frases que se cruzan en ese encuentro inicial:

'Now do come and have this one wi' me', he said caressively. 'It's easy, you know. I'm pining to see you dance'.

[...]

'No, I won't dance', she said softly. Her words came clean and ringing.

[...]

'But you mustn't miss your dance', she reproved. (p. 15)

Como se puede observar, la respuesta de Gertrude a la invitacion del minero no es sólo dista de ser una negativa tajante sino que por su tono y hasta contenido no le hace sentirse rechazado. $Y$ es en ese preciso momento cuando esa cortesía verbal para con ella empieza a dar paso de nuevo a la variante cerrada del dialecto que suele utilizar Walter Morel:

'Nay, I don't want to dance that -it's not as I care about'.

'Yet you invited me to it'.

He laughed very heartly at this.

'I never thought $O$ ' that. Tha'rt not long in taking the curl out of me'.

It was her turn to laugh quickly.

'You don't look as if you'd come much uncurled,' she said.

'I'm like a pig's tail, I curl because I canna help it', he laughed, rather boisterously. (p. 15-6)

No cabe duda de que, consciente del interés y la atracción que suscita, Walter Morel se siente ahora más seguro de sí mismo y reafirmado en su propia habla y cultura. Siente que esa deferencia especial que ha tenido para con ella -y no sólo en la acepción común de esta palabra, sino también en el sentido técnico que el término cobra en el análisis sociolingüístico- ya no es necesaria. Así, mientas en el primer intercambio verbal apenas si se le había "deslizado" el debilitamiento -con respecto al estándar- de la preposición 'with' ('wi'); en el segundo, se aprecia el uso del adverbio dialectal de negación 'nay' y el valor de pronombre relativo que tiene a menudo la forma 'as'. Así pues, este abandono temporal de la variante cerrada del dialecto que suele emplear Walter Morel, en favor de un inglés próximo al utilizado por las clases medias que conviven con su entorno cultural, no es 
sino un acto de cortesía verbal, una clara concesión a la cultura de la joven a la que acaba de conocer.

El contexto de este encuentro, del que surge la futura relación de estos dos personajes, es de sobra conocido y, en todo caso, se deprende totalmente del dialogo. El minero invita a Gertrude a bailar; y aunque ésta no accede no le hace sentirse rechazado, pues le da muestras de que su compañia le resulta grata. Esa actitud de Gertrude hace que se incremente su autoestima, generando una confianza en sí mismo que se manifiesta, como nos dice el narrador, en una explosión de hilaridad ('He laughed very heartly at this $[p, 15]$ ') y en una vuelta inmediata a su habla vernácula. En efecto, en ese instante, precisamente porque el dominio de la situación le hace sentirse más seguro de sí mismo, Walter Morel continúa y se readirma en el uso del dialecto que acababa de iniciar: 'I never thought o' that. Tha'rt not long in taking the curl out of me' (p. 15). La función de ese cambio súbito de codigo, así como la de la "deferencia" sociolingüística que muestra al utilizar un tipo de inglés con el que ni se identifica ni se siente cómodo, queda más que patente en el texto. Esos cambios expresan la alta emotividad del encuentro y hablan de las fuertes barreras que puede superar el amor que siente hacia la joven. No cabe duda, por lo tanto, de que se trata de un momento muy importante de la historia que nos cuenta Lawrence.

A pesar de esa importancia, y aunque la respuesta que los traductores dan a estas frases varía de unos a otros, esa marca de emotividad que entrañan el paso del dialecto al estándar y la posterior vuelta al dialecto se debilita excesivamente en las versiones españolas, y en alguna traducción incluso desaparece por completo. También se da el caso -el peor que se puede contemplar, según se verá- de que ese cambio de variedad o registro y su función en el texto original se perviertan en el texto de llegada.

Así, en la traducción de Luis de la Plaza, la dos frases de Walter Morel más arriba comentadas se transforman en '¡Hombre!, no se me había ocurrido. T'ha faltao tiempo pa' dejarme chafao'6. No parece

6 Lawrence, D.H., Hijos y amantes. Traducción de Luis de la Plaza (Madrid: Alianza Editorial, 1983, p. 19). A partir de ahora todas las referencias textuales a la 
necesario insistir demasiado en la asimetría que existe no sólo entre el léxico de este texto traducido y el del original sino, sobre todo, entre el tono y las connotaciones de ambos segmentos. Baste con decir que en esas dos frases del diálogo original se percibe la impronta del sabor local de esa cultura popular de la que se nutre el habla del minero. Así, un concepto metafórico del gusto de 'taking the curl out of me' se sitúa en las antípodas de una frase tan vulgar como 'me has quedao chafao'. En la obra de partida, aunque Gertrude no ha aceptado del todo su invitación, el lenguaje del minero en ningún momento cae en la vulgaridad. Por el contrario, el tono de sus palabras en el texto traducido, aunque el contexto situacional es el mismo, resulta como mínimo chabacano. Como se ha visto, con el término 'chafao' no sólo no se vierte sino que se pervierte la base metafórica de la hermosa expresión anteriormente citada. Si a eso añadimos la inserción gratuita de la exclamación '¡Hombre!', -término entre coloquial y vulgar- se confirma que las elecciones de esta traducción son precisamente prueba del mal gusto. Por supuesto, lo que se objeta aquí no es la elección de un lenguaje subestándar sino la de la variante concreta a que ha recurrido el traductor. Ésta, según se puede apreciar, se acerca peligrosamente a ciertas jergas semimarginales cuyas señas de identidad oscilan a menudo entre la arrogancia, la ignorancia y la chabacanería más atrevida.

Desgraciadamente no es éste el único pasaje en que el lenguaje del texto original y el del de Luis de la Plaza divergen de manera tan acusada. Recordemos, por ejemplo, el lenguaje de otros episodios de cuya relevancia en el conjunto de la obra cabe muy poca duda, con el fin de valorar cómo se transfiere al español. Uno de ellos es esa conocida parte del relato en que el minero irrumpe en el diálogo que mantienen su esposa y el señor Heaton, el pastor, mientras toman el té7. Se trata

traducción de Luis de la Plaza corresponderán a esta edición y la página se indicará en el texto entre paréntesis.

7 En efecto, esa diferencia de origen social que separa a la pareja afecta a su propia visión del mundo que, si bien en cierto modo puede complementar su unión, también contribuye a separar a los cónyuges. Dimin Luo resume, en un comentario muy pertinente, la dimensión espiritual de esa diferencia: “Mrs. Morel's dream was to have a highly spiritual life, while Mr. Morel provided her a kind of completely realistic life, which is entirely different from Mrs. Morel's morality and religious

Futhark 9 (2014)

Conejero Magro, La función del dialecto de Walter Morel, 65-84

ISSN 1886-9300 
de uno de esos momentos en que la confrontación dialectica entre dos maneras de vivir y entender el mundo alcanza su punto álgido. Veamos, en primer lugar, algunos de los fragmentos mas sustanciosos de la conversacion que se entabla entre los tres en la obra de partida:

He [Walter Morel] nodded a How d'yer do' to the clergyman, who roce to shake hands with him.

Nay', said Morel, showing his hand, look thee at it! Tha niver wants ter shake hands wi' a hand like that, does ter? There's too much picjhaft and shovel-dirt on it.'

[...]

'Are you tired?' asked the clergyman.

'Tired? I ham that', replied Morel. 'You don't know what is to be tired, as I'm tired'.

'No', replied the clerguman.

'Why, look yer 'ere', said the miner, showing the shoulders of his singlet. 'It's a bit dry now, but it's wet as a clout with sweat even yet. Feel it.

'Goodness!' cried Mrs. Morel. Mr. Heaton doesn't want to feel your nasty singlet.'

[...]

'Aven't you got a drink, Missis, for a man when he comes home barkled up from the pit.'

'You know you drank all the beer', said Mrs Morel, pouring out his tea.

'An' was there no more to be got?' Tuming to the clergyman -'A man gets that caked up wi' th' dust, you know, -that clogged up down a coalmine, he needs a drink when he comes home.'

'I am sure he does,' said the clergyman.

'But it's ten to one if there's owt for him.'

'There's water -and there's tea,' said Mrs Morel.

'Water! It's not water as'll clear his throat'

(pp. 42-3).

En estos fragmentos se perciben no sólo las peculiaridades lingüísticas del habla del minero sino también el orgullo con que se refiere a su trabajo. Ese orgullo es un componente esencial de la reafirmación

thoughts[; the] torture of the life broke her dream ... [because] of this distinct social background and the degree of education, they had different philosophies and values" ("Code Switching in Sons and Lovers" en Advances in Literary Study 1. 4. [2013: pp. 39-42], p. 40). 
en su cultura cuyo núcleo es la actividad minera por lo que el uso del estándar resulta estéticamente imprescindible. De ahí que la reducción del discurso dialectal del minero a unas cuantas formas de un español "excesivamente" subestándar, como ocurre en el texto de Luis de la Plaza, entrañe una degradación inadmisible del texto original:

Con un movimiento de cabeza, lanzó un "¿Cómo 'stá usté?" al pastor, que se levantó para darle la mano.

¡Quita allá! -dijo Morel, enseñándole la mano-, mira cómo la tengo. No querrás estrechar una mano como ésta ¿verdad? Apesta demasiado a mango de pico y polvo de pala.

[...]

¿Está usted cansado? -preguntó el pastor.

¿Cansado? ¡Ya lo creo! -contestó Morel-. Usté no sabe lo qu'es estar cansao como lo estoy yo.

No.

¡Mire, mire esto! -dijo el minero, mostrándole las hombreras de su chaleco-. Ya se ha seca un poco, pero aun se podría retorcer como un trapo pa' escurrir el sudor. ¡Toque!

¡Dios mío! -gritó la señora Morel-. El señor Heaton no tiene ninguna gana de tocar tu chaleco asqueroso.

El pastor alargó la mano tímidamente.

No pue' que no -admitió Morel-; pero todo es sudor que me sale del cuerpo, quieras que no. Y ca' día igual, acabo con el chaleco chorreando, ¿yo no ti'e usté algo de beber señora, pa' un hombre que vuelve a casa to' encostrao de la mina?

Ya sabes que te bebiste toda la cerveza -dijo su mujer, sirviéndole una taza de té.

¿Y es que no se podía comprar más? -se volvió hacia el pastor-. Cuando uno se pone así de embardunao de polvo reseco, ¿sabe? Atascao allí en el fondo de una mina de carbón, uno necesita un trago al volver a casa.

Claro que sí- asintió el pastor.

Pero pue' apostarse diez contra uno que no habrá ni gota pa' él.

Hay agua .... y hay té- dijo la señora Morel.

¡Agua! ¡No se pué uno enjuagar el gaznate con agua!

Se llenó el platillo de té, sopló y lo aspiró a través de su bigotazo negro, después de lo cual dio un suspiro. Luego se llenó otra vez el platillo y dejó la taza sobre la mesa.

¡Mi mantel!- soltó la señora Morel, poniendo la taza encima de un plato. 
Cuanko uno vuelve a casa tan rendido como yo, qqué lene le puen importar los manteles! - dijo Morel.

(...)

Senor Heaton-dijo-, cuando un s ha pasao to'l santo dia en el fondo de un agujero, picando veta carbón ... pos si bastante más dura quésa pare ... (pp. 50-1).

La degradación de la calidad literaria a la que se acaba de hacer referencia no es ajena al hecho de que, en esta traducción, las escasas marcas del subestandar de Walter Morel pueden resultarles excesivamente vulgares, $o$ incluso malsonantes, a no pocos lectores de habla española.

A pesar de que, como se acaba de ver, este lenguaje se aleja del original hasta extremos que rebasan el límite de lo admisible, la de la Plaza no es aún la peor de las traducciones. Hay otra, realizada por Esther Belfer de Sanguinetti y publicada por Editorial Freeland ${ }^{8}$, en la que tanto las palabras que intercambian al principio el joven Walter Morel y su futura esposa, como las que acabamos de oír pronunciar a esta última, al clérigo $\mathrm{y}$ al minero mientras toman el té se funden y hasta confunden en un nivel de habla idéntico. Es decir, en esa version, las diferencias culturales y lingüísticas tan marcadas y que con tanta nitidez caracterizan a Walter y a Gertrude Morel los personajes, se diluyen en un español estándar idéntico para todos. He aquí el texto de la conversación en la traducción de esta autora:

Murmuró un “¿Cómo está Ud.?” Al clérigo, que se levantó para estrechar su mano.

No -dijo Morel, mostrando su mano-. ¡Mire aquí! Esta nunca estrechará una mano como ésta. Hay demasiados rastros del pico y demasiada suciedad en ella.

[...]

¿Está cansado? -preguntó el clérigo.

¿Cansado? Eso es poco -replicó Morel-. Usted no sabe lo que es estar cansado, como lo estoy yo.

No -dijo el clérigo.

${ }^{8}$ Lawrence, D.H., Hijos y amantes. Traducción de Esther Belfer de Sanguinetti (Buenos Aires: Editorial Freeland, 1960). A partir de ahora todas las referencias textuales a Hijos y amantes corresponderán a esta edición y la página se indicará en el texto entre paréntesis.

Futhark 9 (2014)

ISSN 1886-9300 
Mire aquí -dijo el minero, mostrando los hombros de su ropa-. Está sucio ahora, pero está mojado en sudor. Siéntalo.

¡Por Dios! -gritó la señora Morel-. El señor Heaton no quiere tocar tu sucio uniforme.

El clérigo retiró su mano escrupulosamente.

No, tal vez no quiera -dijo Morel-. Pero es así, le guste o no. Un día de trabajo deja mi ropa chorreando sudor. ¿No tiene un trago, señora, para un hombre que viene a su casa cansado del trabajo en la mina?

Sabes que te tomaste toda la cerveza -dijo la señora Morel, sirviéndole el té.

¿Y no puede conseguirse más? -Volviéndose al clérigo: Cuando un hombre se ha pasado todo el día bajo tierra, hundido en una mina de carbón, necesita un trago cuando llega a casa.

Estoy seguro de que lo necesita -dijo el clérigo.

Pero generalmente no hay nada para él.

Hay agua ... y hay té - dijo la señora Morel.

¡Agua! No es agua lo que aclara mi garganta.

Llenó una taza de té, lo sopló, y lo sorbió a través de su gran bigote negro, suspirando después. Luego se sirvió otra taza, y la puso sobre la mesa.

¡Mi mantel! -dijo la señora Morel, colocándola sobre el plato.

Un hombre que llega a su casa como yo, está demasiado cansado para preocuparse por el mantel -dijo Morel.

[...]

Señor Heaton -dijo-, un hombre que ha estado todo el día allá abajo, en ese agujero negro, llenándose la cara de carbón, con la respiración más dura que esa pared ... (pp. 41-3).

Como se puede comprobar, con tan precaria y torpe respuesta esta traductora destruye una parte esencial de la riqueza de la obra, que es ante todo una riqueza verbal, privando al lector de lengua española del exponente estilístico más fehaciente de la tensión que dinamiza el relato de Sons and Lovers $^{9}$. De la Plaza al menos reflejaba lingüística-

9 Sobre la importancia del dialecto en la caracterización de Walter y su centralidad en la obra, coinciden prácticamente todos los estudios en que se trata el tema, aun cuando diverjan en su enfoque o interpretación de la novela. En ese sentido, la afirmación que hace E. P. Shrubb reviste especial interés: "[The] language in which [Walter Morel] criticizes his wife's determination to keep William out of the pit is, surely, part of what saves him. It preserves him in the world of the working class, saves him, for one thing, from earning the sort of moneyin the sorts of conditions that free William to try for the things that money in the city is invited by it to try

Futhark 9 (2014)

Conejero Magro, La función del dialecto de Walter Morel, 65-84

ISSN 1886-9300 
mente la existencia de un conflicto, aunque la variante sociolectal elegida diste mucho de ser la adecuada. Pero con la traducción de Belfer se pierde la esencia misma de esa tensión, con lo que la calidad literaria de la traducción se resiente sobremanera.

En consecuencia, ambas traducciones, si bien en diferente medida, se desvian de la senda por la que debieran discurrir. De ahí quizá que la tónica de las siguientes intervenciones del minero en ambos textos de llegada apenas varie con respecto a la de las anteriores. Fruto de esa elección, de una variedad no estándar inadecuada, en la versión de de la Plaza no se aprecia sino un dialogo excesivamente degradado; $\mathbf{y}$ en la de Belfer, el resultado de esa dilución del vivo contraste lingüístico del original, que impide que se asome cualquier rasgo de dialecto regional o social, no es sino la ya aludida mutilación estilística que afecta a la totalidad de la obra.

El tratamiento del resto de los diálogos en que interviene Walter Morel corrobora esa degradación y mutilación a que de la Plaza y Belfer, respectivamente, someten a la obra. Así, en la traducción de de la Plaza, un fragmento tan marcado por rasgos dialectales como

'What dost want ter ma'e a stool-harsed Jack on 'im for?' said Morel. 'All he'll do is to wear his britches behind out, an' earn nowt. What's 'e startin' wi'?' (p. 62)

se transforma en un par de oraciones salpicadas de alguna que otra característica del tipo de subestándar anteriormente indicado:

- ¿Y pa' qué quiés hacer d'él un chupatintas? -dijo Morel-. No hará más que desgastarse el fondo sus pantalones, y de ganar, na. ¿Cuánto le dan pa'empezar? (p. 74);

y en la de Belfer, al no dar esta autora respuesta alguna al problema del dialecto, las palabras de Walter Morel se tranforman en espanol estándar:

for. His language, also, is a language of men; it is note ven the language his mother speaks, which, like the snippets we hear from other working-class women in the novel, is not vivid, but only ill-educated." ("Reading Sons and Lovers" en Sydney Studies in English, 6. [1980-1981: pp. 87-99], p. 95). 
- ¿Para qué quieres hacer de él un trabajador de taburete? -dijo Morel- Todo lo que hará será acostumbrarse mal, y no ganará nada. ¿Con cuánto está empezando? (p. 62).

Al menos de la Plaza capta mejor ese sentido peyorativo que Walter Morel da a la expresión '[to be] a stool-[arsed] Jack', que por cierto híper-corrige añadiendo un sonido fricativo glotal sordo ([h]). En efecto, 'chupatintas', la formula de de la Plaza, es una expresión más proxima al original y, por lo tanto, afortunada que la de Belfer: 'trabajador de taburete'.

Un enfoque muy parecido aplican ambos traductores a las siguientes palabras que pronuncia Walter Morel en un momento de gran enfado:

'If tha opens it again while I'm weshin' me, I'll ma'e thy jaw rattle,' he threatened from the midst of his soapsuds. Paul and the mother frowned to hear him (p. 225).

La reducción excesiva de las marcas del subestándar, en la versión de de la Plaza, difumina aún más los contornos del habla de Walter Morel y de la de su esposa y a menudo sus hijos:

-Si te s'ocurre abrirla otra vez mientras que m'estoy lavando, te parto los morros - amenazó, mientras seguía enjabonándose. Paul y su madre fruncieron el ceño al oírlo (p. 260).

Por esa proximidad al estándar, en esta ocasión el perfil verbal de Walter Morel se asemeja al de la traducción de Belfer:

- Si eso sucede nuevamente mientras me estoy lavando, te haré sonar la mandíbula - amenazó en medio de la espuma del jabón. Pablo y su madre se fruncieron al escucharlo (p. 226).

No obstante, la traducción libre que de la Plaza propone para '[to make your] jaw rattle' ('te parto los morros') se acerca mucho más a la expresión original de partida que la traducción casi literal de Belfer ('te haré sonar la mandíbula').

Por último, tampoco coinciden de la Plaza y Belfer en otro fragmento marcado por el casticismo y la vena popular que recorre el habla del minero: 
'Like a moudiwarp!', he laughed. 'Yi, an' there's some chaps as does go round like moudiwarps' (p. 16).

En este caso, de la Plaza prescinde por completo de cualquier marca subestándar en el habla de Walter Morel, por lo que esta queda desprovista de cualquier traza dialectal: " - „Como un topo! -se rió él¡Y si que hay algunos tíos que andan como topos!-" (p. 20). Sin embargo, como se puede ver, el significado de estas dos frases nos depara esa nota castiza que su significante nos niega: el símil feliz entre el topo y el minero. En cuanto al texto de Belfer, la falta de coincidencia se debe a que esta autora opta, sin razón alguna, por prescindir de estas dos frases en su traducción.

Conviene insistir en que la función principal del habla de Walter Morel, aún estando impregnada de ese sabor peculiar y propio de su tierra, tiene un sentido más radical. Con ella reafirma su personalidad y los valores del trabajo de la mina, es decir, de la clase obrera, que es la "suya", frente a ese mundo "artificial" y, para él, "ocioso" de los "otros". Estamos ante una metáfora de la lucha de clases, de la dialéctica "us"/"them", términos que tradicionalmente han expresado esta pugna en los medios obreros británicos. "Them" son los que hacen un trabajo físico, los oficinistas de la empresa (los 'chupatintas'), el maestro, el clérigo y, para mayor desgracia de Walter Morel, su propia esposa y hasta sus hijos. En medio de este ambiente totalmente hostil, pues no halla sosiego ni en su propia casa, Walter Morel parece aferrarse a su propio dialecto como expresión de todos sus valores. Este es el sentido principal de su uso del habla de Nottinghamshire en Sons and Lovers, aunque se complete y complemente con lo anteriormente referido. Pues bien, gracias precisamente a esa doble función del dialecto en esta obra, no estamos ante un intraducible absoluto, es decir, su versión a otra lengua no es del todo imposible.

Conviene dejar esto muy claro, aun cuando resulte reiterativo, pues si su función se redujese únicamente a la adición de color y sabor local o, lo que es lo mismo, si el dialecto de Walter Morel tuviera un papel meramente folclórico o de transmisor de la cultura regional, estaríamos ante un intraducible, ya que, como se indicaba anteriormente, no es posible encontrar un dialecto de resonancias locales y connotaciones culturales idénticas en otras lenguas, pues no existe. 
Esto quiere decir que, en el caso del habla del minero, tan sólo se puede aspirar a reproducir en el texto de llegada esas marcas sociolinguísticas que señalan su condición social $y$, sobre todo, la dialéctica social en que se halla inmerso. Intentar el traslado de cualquier otro rasgo será siempre un empeño vano. Esta conclusión coincide en parte con una de las reflexiones que hace Inés Praga Terente en un trabajo sobre la traducción de esta misma obra ${ }^{10}$. En concreto, al considerar la hipótesis de la utilización del bable para el traslado del dialecto de Lawrence, pues a primera vista podría parecer el más idóneo para ocupar el lugar del habla también minera de Nottinghamshire, esta autora afirma lo siguiente:

la adopción del criterio de Catford' [que consiste en seleccionar un dialecto equivalente en lengua de llegada] 'significaría un dato negativo para la traducción de Sons and Lovers. La inserción del personaje de Walter Morel en un contexto asturiano resultaría harto artificiosa11.

En efecto, el bable en boca del minero inglés resultaría ridículo aquí, pues, como se ha indicado de modo reiterado en las páginas precedentes, no sólo no hay equivalencia sino ni siquiera parecido entre dos lenguas que se han desarrollado en espacios culturales tan distintos y distantes. Por eso el traductor debe descartar esta fórmula, por atractiva que resulte la coincidencia de que la minería e incluso la cultura minera ocupen un lugar destacado en las regiones en que se hablan tanto el dialecto en que se inspira Sons and Lovers como el bable.

También hay que proceder con suma cautela con respecto a otra de las propuestas que se han hecho para solucionar el problema, pues se puede correr el riesgo de producir un efecto contrario al perseguido. Se trataría, en concreto, de la opción por un idiolecto para caracterizar verbalmente al personaje minero. Praga Terente, que defiende esta solución en el referido artículo, propone

10 Praga Terente, Inés, "Algunas consideraciones sobre la traducción al castellano de Sons and Lovers de D.H. Lawrence", ES, 13 (septiembre, 1983), Valladolid: Universidad de Valladolid, pp. 185-202.

${ }^{11}$ Praga Terente, Inés, op.cit., p.194.

Futhark 9 (2014)

Conejero Magro, La función del dialecto de Walter Morel, 65-84

ISSN 1886-9300 
crear en la traduccion un idiolecto particular para Walter Morel, cuidando de que éste se ciña escrupulosamente al personaje'; y anade: 'de este modo, deberian analizarse con todo rigor sus características (incultura, temperamento sangúneo, espontaneidad, facilidad de comunicación etc.) para incluir en su discurso aquellos elementos que se correspondan con éstas (vulgarismos, sintaxis simple, elipsis) y excluir otros (cultismos, construcciones artificiosas, etc.) ${ }^{12}$.

El peligro de esta propuesta se halla en el hecho de que al singularizar verbalmente a Walter Morel de esta manera, es decir, al marcar fuertemente su habla con unas características 'individuales', se podría producir una separación y un aislamiento aun mayores del personaje, pues se le alienaría también de ese grupo social al que tantos y tan fuertes lazos le unen. Se le negaría, en la estructura argumental de llegada, el único refugio que Lawrence le reserva en el texto original: el medio cultural de su clase. Pues, si bien es cierto que el uso del dialecto le separa de ese mundo "superior" y hostil que se expresa en estándar, al menos le cobija y protege, integrándole en la cultura local y el entorno sociolingüísticos en que vive. Sin embargo, al cargar en exceso las tintas sobre el idiolecto, se corre el riesgo, como se acaba de decir, de divorciar al personaje incluso de quienes participan de su sistema de valores y modo de vida. Es decir, la diferencia entre recurrir al dialecto y al idiolecto es la misma que existe entre la afirmación de una identidad individual y la de la pertenencia a una clase que, en este caso, es tanto como decir a una cultura o una subcultura, si se quiere, que es heredera de una tradición marcada por un modo de vida distinto y una escala de valores propia.

En resumen, al trasladar su lenguaje a otro idioma, siempre se perderá gran parte de ese componente cultural de los East Midlands que con tanta fuerza se proyecta en Sons and Lovers. No obstante, si descartamos el recurso al dialecto regional y al idiolecto, y buscamos una correspondencia del habla del minero en una variante subestándar de la lengua de llegada -pero sin caer en la chabacanería o la vulgaridad de la versión de Luis de la Plaza-, podremos recrear una parte también importante de la idiosincrasia del minero, esas señas de identidad que certifican su pertenencia a un grupo. La solución, pues,

12 Ibid. 
hay que buscarla en un tipo de lengua que, aun situándose por debajo del español normal (entendiendo el término 'normal' en el sentido de la norma linguística general, y el prefijo 'sub-' en el de su posición en el eje vertical), no esté exenta de esas expresiones idiomáticas o giros que dan al texto inglés original la viveza que exhiben a menudo las intervenciones del minero inglés.

En consecuencia, parece que con la versión del dialecto inglés a un español subestándar, pero no empobrecido en cuanto a la imagen verbal o a la expresión, se puede garantizar al menos la reproducción de ese conflicto social y cultural en que está inmersa la familia de Sons and Lovers y que acabará dividiéndola y destruyendo su convivencia. En efecto, en mi opinión, este tipo de lengua puede marcar lingüísticamente al Walter Morel de la versión española, expresando y diferenciando claramente su identidad con respecto a un mundo más culto y dominante. Por eso la utilización de una variante de dialecto social de este tipo en la traducción parece la respuesta más acertada en este caso. A su vez, la expresión de ese mundo de la clase y cultura dominantes se lograría poniendo un castellano más refinado en boca de su esposa y de los personajes que comparten o imitan su modalidad lingüística. Así, en la traducción de esta obra, un sociolecto sería la fórmula más próxima a un geolecto o dialecto geográfico. Para utilizar una formulación muy común en la sociolingüística tradicional, el habla del señor Morel, en el texto de llegada, se situaría únicamente en el eje vertical del lenguaje, y en ningún caso en el horizontal. Aún así la versión española perderá mucho de ese acervo cultural que entraña el habla de Morel, pero al menos no le marginará ni le alejará de su comunidad ${ }^{13}$. Dicho de otro modo, ya que el dialecto regional desempeña también toda una función de diferenciación social en

${ }^{13}$ Incluso con esta fórmula -tan precaria desde la perspectiva del sabor local y los valores culturales del geolecto-, la traducción podría exhibir esa versatilidad lingüístico-estilística que Oxana Ruxandra Hrițcu reconoce como fundamental en Sons and Lovers: "The world of Lawrence's fiction os one that can at any time be challenged by the narrator himself, who is capable of exploring and adopting any style of language that would seem to be at odds with the normal conventions of English fiction." ("Multiple Levels of Verbal Expression and Character Portrayal: A Study on D.H. Lawrence's Sons and Lovers", en Cultural and Linguistic Communication 2. 3. Duly/September 2012: pp. 235-41], p. 241). 
el entramado de Sons and Lovers, y teniendo en cuenta que el sabor local, al igual que la poesía más íntima, es un intraducible, puede que éste sea ese mal menor que, en el arte de la traducción, supone con frecuencia el límite de lo posible.

\section{BIBLIOGRAFÍA}

BALbERT, Peter. D. H. Lawrence and the Phallic Imagination: Essays on Sexual Identity and Feminist Misreading. Hong Kong: Macmillan, 1989.

CARbonell i CORTÉs, Ovidi. Traducción y cultura, de la ideología al texto. Salamanca: Ediciones Colegio de España, 1999.

CATFORD, John C. A Linguistic Theory of Translation. Oxford: Oxford University Press, 1967.

HERVEY, SÁNDOR, E IAN HIGGINS. Thinking Translation. London: Routledge, 1992.

INGERSOLL, Earl. "Gender and Language in Sons and Lovers." Midwest Quarterly 37.4 (1996): pp. 434-47.

LAVOB, William. Sociolinguistic Patterns. Philadelphia: University of Pennsylvania Press, 1972.

LEITH, Richard. "Dialogue and Dialect in D. H. Lawrence." Style 14 (1980): pp. 245-258.

NeWmark, Peter. Approaches to Translation. Hertfordshire: Prentice Hall International, 1988.

RABADÁN, Rosa and Trinidad Guzmán. "Las inequivalencias lingüisticas en la traducción inglés-español." Translation Across Cultures. La traducción entre el mundo hispánico y el anglosajón. XI Congreso de AEDEAN. Ed. J. C. Santoyo. León: Universidad de León, 1989. pp. 141-145.

WILSON-LEWIECKI, Cynthia. Writing Against the Family: Gender in Lawrence and Joyce. Carbondale: Southern Illinois UP, 1994.

Futhark 9 (2014)

ISSN 1886-9300

Conejero Magro, La función del dialecto de Walter Morel, 65-84 